\title{
Propuesta de Desarrollo para el Turismo de Aventura en el territorio de la Llanura Habana-Matanzas en los límites de las provincias La Habana y Mayabeque
}

Proposal on the development of adventure tourism based in the HavanaMatanzas plain between the limits of Havana and Mayabeque provinces

Claudia Saumell Lafargue ${ }^{1}$ \& Gerardo Carlos Hernández Rodríguez ${ }^{2}$

\begin{abstract}
.
The present article constitutes a Proposal on the Development of Adventure Tourism based in the Havana-Matanzas Plain between the limits of Havana and Mayabeque provinces. The Research on thetourist resources in this territory, allowed for the selection of the potential ones for the enhancement of the Modality. San José de Las Lajas y Melena del Sur, in Mayabeque province were determined to comprehend the area. In correspondence with its physical geographic characteristics, together with a market Research and tendencies of this type of Tourism, a proposal was designed, with the creation of tourist products. By the Expert Criteria, the ones that have better chance to be succesfull were known. The Opportunity Study of the products was made.
\end{abstract}

Keywords: Havana-Matanzas Plain, Adventure Tourism, Melena del Sur, San José de Las Lajas, Develpoment, tourist product.

${ }^{1}$ Grupo Hotelero Cubanacan, La Habana, Cuba. claudia.saumel196@gmail.com

${ }^{2}$ Universidad de La Habana, La Habana, Cuba. gerard.chr97@gmail.com 


\section{Resumen.}

El presente artículo constituye una Propuesta de Desarrollo del Turismo de Aventura en la zona de la Llanura Habana-Matanzas enmarcada en los límites de las provincias La Habana y Mayabeque. El estudio de los recursos turísticos existentes en dicho territorio, permitió la selección de los que constituyen potenciales para el desarrollo de productos de dicha modalidad. Se determina que la zona a desarrollar está compuesta por los municipios San José de Las Lajas y Melena del Sur, en la provincia de Mayabeque. En correspondencia con las características físico-geográficas de la misma y, unido a un estudio de mercado y tendencias de esta tipología de Turismo, se diseña una Propuesta de Desarrollo de la Modalidad, a partir de la creación de productos turísticos. Basado en los criterios de expertos, se determinan los que poseen mayor probabilidad de éxito. Se realiza el Estudio de Oportunidad de los productos.

Palabras claves: Llanura Habana-Matanzas, Turismo de Aventura, Melena del Sur, San José de las Lajas, Desarrollo, producto turístico.

\section{Introducción.}

A lo largo de las últimas décadas, el turismo ha experimentado expansión y diversificación continuas, convirtiéndose en uno de los mayores y más crecientes sectores económicos en el mundo. Este, en sentido general, afronta cambios continuos como consecuencia de la situación económica a nivel mundial. La crisis afecta directamente el flujo turístico y, por ello, las ofertas y los destinos se enfrentan a un entorno altamente competitivo. Resulta entonces importante lograr la satisfacción de los clientes y brindar productos adecuados a sus deseos y motivaciones, así como aprovechar las potencialidades de cada destino para ello.

Sobre el caso cubano, (Lorenzo, 2016) afirma que el incremento progresivo de turistas en la Isla, se ha centrado fundamentalmente en el modelo sol y playa y ha comportado evidentes impactos negativos a nivel de patrimonio natural por la propia naturaleza de esta tipología turística, que no puede generalizarse si queremos conservar la biodiversidad y los paisajes cubanos autóctonos. Ya desde estos años, los organismos gubernamentales encargados de la gestión del Turismo, detectaron la necesidad de diversificar el sector e intentar cambiar la imagen del destino. Desde el momento que se empezó a detectar el declive del producto todo incluido en el destino, así como la carencia en la diversidad de los mismos, se empezaron a plantear políticas y esfuerzos que fueran encaminados a dicha diversificación de Cuba como destino turístico.

Se hace necesario enfocar los esfuerzos en la ampliación de la oferta en un destino altamente competitivo, con recursos turísticos que aún no han sido explotados. En este sentido, se han desarrollado investigaciones para actividades clave como la concepción de los recursos 
existentes y factibles a explotar; sin embargo, es necesario que se haga un análisis más profundo que tenga como principal objetivo conocer la situación de las áreas y su potencial para el desarrollo turístico. La continuidad de estos estudios resulta primordial para el avance del Turismo de Aventura, como forma de diversificar la oferta turística en el país, a una escala superior de importancia y como representativo de la actividad en el destino Cuba. Se continúa desaprovechando posibilidades de crecimiento económico asociado a los recursos naturales.

Para un mejor entendimiento y homologación de conceptos, se realiza un análisis profundo sobre las modalidades y sub-modalidades turísticas. Se toma entonces, como referencia, la Propuesta de la Secretaría de Turismo (SECTUR) en el año 2016, siendo este que el turismo de naturaleza comprende el ecoturismo, turismo de aventura y trismo rural.

Cualquier actividad turística que requiera la transformación dura del espacio o la creación de instalaciones y de determinados elementos mecánicos no solo como apoyo a la estancia (alojamientos, sistema de transporte, etc.), sino como factor indispensable para la práctica, puede realizarse en la naturaleza; sin embargo, no sería motivada por esta de forma directa. De esta forma, no podrán considerarse como actividades de Turismo de Naturaleza (Barrado \& Vila, 1999). En realidad, la naturaleza no es más que un mero contenedor que se limita a acoger de forma pasiva una serie de usos en los que lo verdaderamente importante, en última instancia, no son los recursos naturales o el paisaje; sino la calidad de las instalaciones y servicios imprescindibles para la práctica deportiva. Sobre este estudio, se aclara entonces que se tomarán en cuenta las actividades que no impliquen una transformación física del medio, sino aquellas en las que los turistas utilicen medios en el espacio natural.

Según Gaceta Oficial de la República de Cuba, No. 25, Extraordinaria, del 2014, el Turismo de Aventura se entiende como el conjunto de las actividades turísticas que implican determinado esfuerzo físico para cumplimentar el reto y el desafío, con el uso de equipamiento o no y sobre la base del riesgo controlado y la seguridad del turista, sin degradar o agotar los recursos y el medio ambiente.

Los principales retos que enfrenta el país en relación al desarrollo del Turismo de Aventura son: la existencia de recursos inutilizados y la infraestructura turística enfocada a los productos de sol y playa como prioridad dentro del Turismo de Naturaleza.

En este sentido, se plantea el siguiente Objetivo General:

Diseñar una Propuesta de Desarrollo de Turismo de Aventura, para el territorio seleccionado de la Llanura Habana-Matanzas.

Para dar cumplimiento al objetivo general se derivan los siguientes objetivos específicos: 
1. Fundamentar los referentes teóricos sobre el Turismo de Aventura como Modalidad Turística.

2. Establecer el procedimiento metodológico de la Investigación.

3. Describir el desarrollo y potencial del Turismo de Aventura en Cuba y en el territorio seleccionado.

4. Elaborar el Proyecto de Desarrollo para la zona a explotar.

Se arriba entonces a la siguiente Hipótesis:

La Propuesta de Desarrollo del Turismo de Aventura en el territorio seleccionado de la Llanura Habana-Matanzas contribuirá a convertirlo en un destino de referencia de la Modalidad en Cuba.

La presente investigación resulta importante pues amplía la imagen de Cuba como destino turístico. El nicho de mercado de sol y playa refrescaría su imagen asociada a hotel Todo Incluido al ser esta una propuesta diferente. Constituye un criterio de creación de productos importante, la sostenibilidad que implican, lo cual es un atributo esencial en cuanto a las tendencias del turismo y, por consiguiente, la decisión de compra. Se fortalece un submodalidad incipiente en la Isla y se explotan recursos naturales inutilizados en el territorio nacional.

\section{Metodologia.}

La presente investigación se clasifica como cualitativa, pues esta resulta un estudio intensivo y de profundidad para interpretar el fenómeno que se desea. A este tipo de investigación le interesa lo particular; lo contextual, los relatos vividos. En esta, según (Mezquita \& Fiallo Rodríguez, 2002) predomina el Método Deductivo. En correspondencia con la misma bibliografía, en cuanto a su dimensión cronológica, la investigación tiene un carácter descriptivo. Los principales métodos teóricos y empíricos en los que se apoya son:

Inductivo-Deductivo: se obtienen los elementos básicos para la evaluación del estado actual de desarrollo de la modalidad en Cuba, una vez se hayan deducido los principales elementos a tener en cuenta; asimismo, permite sentar las bases para el diagnóstico de su nivel de desarrollo.

Analítico-Sintético: permite identificar el segmento de demanda objetivo para la Propuesta.

Histórico-Lógico: posibilita comprender el comportamiento de los mercados, el análisis de las tendencias y preferencias de los mismos, así como el desarrollo del Turismo de Aventura en Cuba en sus diferentes etapas de existencia para comprender el estado actual. 
Análisis Bibliográfico y Documental: La bibliografía seleccionada contribuyó a crear las bases teóricas y la recopilación de datos para la etapa de campo.

Entrevista: Se realizó a especialistas de las agencias Cubatur, ECOTUR S.A., Gaviotatour S.A., así como de la Empresa Nacional para la Protección de la Flora y la Fauna, con el objetivo de conocer en profundidad el estado actual del Turismo de Aventura en Cuba.

Ciclo de Vida del Producto: Se utilizó para conocer la etapa en la que se encuentra el Turismo de Aventura en Cuba y en el territorio seleccionado. Se describen las etapas de (Barrios, 2007): introducción del producto, una etapa de crecimiento, una etapa de madurez, una etapa de declinación.

Matriz DAFO: Nos permitió tomar decisiones estratégicas a partir de las fortalezas, oportunidades, debilidades y amenazas que se detecten en el análisis interno y externo del territorio en cuestión.

Metodología de Desarrollo y Lanzamiento de Productos Turísticos:

Se realiza una valoración de diversas propuestas que definen los procedimientos para diseñar productos turísticos, unas se enfocan más en el diseño de productos comercializables, pero sin determinar la necesidad de un diagnóstico previo y otras no definen cómo estructurar el producto. Las fuentes principales fueron: (Mincetur, 2014), (IICA Costa Rica, 2018), (Perelló, 2001). El presente trabajo se basa en las diferentes bibliografías consultadas, incluyendo las enfocadas en la elaboración de productos turísticos específicos, así como otras más abarcadoras como la Metodología para la elaboración de un Plan de Desarrollo Turístico en un territorio. El esquema de esta última propone una adaptación de la Metodología para el Desarrollo y Lanzamiento de Productos Turísticos (Atractivos y Recursos) de Ramón Martín Fernández del Centro de Estudios Turísticos de la Universidad de La Habana. La Base Metodológica del mismo establece los siguientes niveles: Nivel I: Concepto y Diseño; Nivel II: Estudio de Oportunidad; Nivel III: Pre-factibilidad; Nivel IV: Factibilidad. La presente propuesta abordará los dos primeros niveles. Para los dos siguientes, serán sentadas las bases para el desarrollo de los trabajos posteriores. Cada nivel incluye sus Fases respectivas, que serán abordas para el desarrollo del producto y las fundamentales serán incluidas en el presente artículo.

\section{Inventario de atractivos turísticos:}

Se realizó el inventario de atractivos turísticos. Para ello, se toma como referencia el "Manual para la Formulación del Inventario de Recursos Turísticos a Nivel Nacional" de MINCETUR: 2006, de la Dirección Nacional de Desarrollo Turístico en Perú. Este establece la agrupación de cinco categorías de recursos turísticos, teniendo en cuenta las características 
propias del potencial turístico peruano: sitios naturales; manifestaciones culturales; folclore; realizaciones técnicas, científicas y artísticas contemporáneas, y acontecimientos programados. Este inventario comprende mayor alcance que el que requiere el presente proyecto, puesto que propone un Levantamiento de Inventario de Recursos a Nivel Nacional; sin embargo, está basado en un sistema de fichas que se ajustan perfectamente con el objetivo de investigación en dicha fase del proyecto. En correspondencia con el objeto del trabajo, el cual no se centra en desarrollar un Destino, sino una Modalidad, como marco de estudio, se incluirá la investigación de todas las categorías de recursos en el territorio, más se elaborará la ficha de inventario solo para los Recursos potenciales de la categoría sitios naturales. De estos se seleccionarán los que constituirán base para los productos a diseñar.

\section{Resultados.}

Sobre las provincias objeto de estudio, los entrevistados no refieren ninguna infraestructura creada o proyecto vinculado al desarrollo del Turismo de Aventura, pues afirman que el desarrollo de esta es incipiente en el territorio. Sobre la comercialización de los productos turísticos, las opiniones coinciden en destacar que la imagen de Cuba está asociada al Turismo de sol y playa, la cual se ha desarrollo de una manera muy exitosa en el país. Los encargados de la comercialización de los productos más novedosos de Turismo de Naturaleza, ecoturísticos en su mayoría, han sido las agencias de viajes convencionales que han abierto su abanico de productos; no obstante, el éxito de esta comercialización no ha tenido los resultados esperados. Se manifiesta, en algunos casos, el poco interés por parte de turoperadores y agencias de viajes en ofrecer este tipo de paquetes debido a que su especialización se centra en el turismo masivo. La primera impresión que se tiene cuando se visita la página web oficial de Turismo de Cuba, es que no se dirige o identifica con una tipología de turismo en específico; resulta muy general. En el único apartado de la web donde se puede encontrar información referente a las actividades es el de destinos turísticos. Entre las actividades que se promocionan son: paseos a caballos, senderismo, observación de fauna y flora, fotocaza y cicloturismo.

\section{Ciclo de vida del turismo de aventura.}

En el Destino Cuba, según datos revelados por el MINTUR y contenidos en (HOSTELTUR, 2016), Cuba aún no explota el $85 \%$ de su potencial turístico, por lo cual el MINTUR ha elaborado un proyecto con perspectivas al 2030, entre cuyas premisas incluye precisamente, el desarrollo del Turismo de Naturaleza, y a fecha de hoy, se hace patente la existencia de productos exitosos de esta tipología. Con esto, se puede decir que el turismo de Aventura en Cuba ha superado su fase de Introducción, puesto que sí se comercializa. Los esfuerzos para las mejoras en la distribución del producto se evidencian con los proyectos para la inserción de pasarelas de pago en algunas agencias. Sin embargo, el Turismo de Aventura aún no se destaca fuertemente en el mercado, pues las acciones de Marketing giran en torno al Turismo 
de Naturaleza en general. Se dilucida entonces que la Modalidad en Cuba se encuentra en la Fase de Crecimiento, pues ya se comprobó la aceptación de dicho tipo de producto en el mercado y se hace necesaria la Promoción más enfocada. Para responder a la demanda y mantener el crecimiento del producto, se debe aumentar la Inversión. En el territorio seleccionado, se ha evaluado el desarrollo del Turismo de Aventura en Cuba, en aras de insertar los productos que se proponen en el presente trabajo, en el marco competitivo del destino. Sobre el territorio seleccionado, las actividades de Turismo de Aventura están vinculadas a los deportes náuticos, sin embargo, los esfuerzos del sector no son suficientes para determinar que esta se encuentre en su etapa de crecimiento; por tanto, se puede concluir que el Turismo de Aventura en el territorio seleccionado de la Llanura Habana Matanzas se encuentra en su etapa de Introducción.

Tabla 1. Matriz DAFO del turismo de aventura en la llanura habana-matanzas

\begin{tabular}{ll}
\hline \multicolumn{1}{c}{ Fortalezas } & \multicolumn{1}{c}{ Dactores Internos } \\
Debilidades \\
$\begin{array}{l}\text { Los paisajes de la naturaleza del } \\
\text { terrirotio se encuentran en estado } \\
\text { optimo de conversación. }\end{array}$ & $\begin{array}{l}\text { Ausencia de caracteristicas físico- } \\
\text { geograficas necesarias para el } \\
\text { desarrollo de algunas actividades } \\
\text { de Turismo de Aventura }\end{array}$ \\
El territorio posee de Recursos \\
Naturales
\end{tabular}$\quad \begin{aligned} & \text { Recursos económicos escasos del } \\
& \text { Gobierno Local } \\
& \text { Imagen consolidada de las zonas } \\
& \text { del territorio en dos ejes } \\
& \text { fundamentales, La Habana para el } \\
& \text { turismo Cultural y de Ciudad y } \\
& \text { Santa Cruz del Norte para Turismo } \\
& \text { de Naturaleza, como competencia } \\
& \text { más cercana. }\end{aligned}$

\section{Factores Externos}

Oportunidades

Amenazas

La situación post-Covid, supone una oportunidad para incremento Posible deterioro de los Recursos muy notable de la llegada de Naturales turistas.

Se ha aprovechado un bajo por ciento del potencial natural en Infraestructura Técnica insuficiente Cuba, reconocido en el esquema para sostener el mantenimiento de de ordenamiento territorial, que productos turisticos y el flujo de guia el desaroolo de la nación con turistas. un enfoque de alta sostenibilidad. 
Incompatibilidad de las

Los viajes de Aventuras características físico-geográficas continúan emergiendo como una del territorio a desarrollar con los de las modalidades más requerimientos físico-geográficos demandadas.

de algunas de las actividades de

Aventura demandadas.

\begin{tabular}{|c|c|c|c|c|c|c|c|c|c|}
\hline 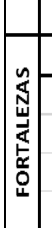 & \multicolumn{5}{|c|}{ OPORTUNIDADES } & \multicolumn{4}{|c|}{ AMENAZAS } \\
\hline \multirow{5}{*}{ 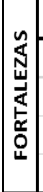 } & & 1 & 2 & 3 & & 1 & 2 & 3 & \\
\hline & 1 & 3 & 3 & 3 & & 3 & 2 & 2 & \\
\hline & 2 & 3 & 3 & 3 & & 3 & 2 & 2 & \\
\hline & 3 & 3 & 3 & 2 & & 2 & 2 & 1 & \\
\hline & & & & & 26 & & & & 19 \\
\hline$\ddot{\omega}$ & \begin{tabular}{|lll}
1 & & \\
\end{tabular} & 1 & 1 & 2 & & 1 & 3 & 0 & \\
\hline 品 & 2 & 2 & 3 & 2 & & 1 & 3 & 0 & \\
\hline$\overline{\mathrm{A}}$ & 3 & 1 & 1 & 2 & & 1 & 1 & 0 & \\
\hline 㟒 & & & & & 15 & & & & 10 \\
\hline
\end{tabular}

Fuente: Elaboración propia.

A partir de la información facilitada por los especialistas y el análisis de diferentes fuentes que se refieren a la situación actual del Turismo de Aventura y del territorio seleccionado, se pudieron determinar los siguientes factores internos y externos que inciden en el desarrollo del Turismo de Aventura en la Llanura Habana-Matanzas.

Luego de realizada la Matriz de Impactos Cruzados, se puede apreciar que las Fortalezas más relevantes para el Turismo de Aventura en el territorio son: la conservación y riqueza de recursos naturales, lo que permite aprovechar el incremento de la llegada de turistas; la explotación de recursos inutilizados y el aumento de la demanda del Turismo de Aventura. Estos factores permiten contrarrestar el deterioro de los recursos naturales, la infraestructura técnica insuficiente y la incompatibilidad de las características físico-geográficas para el desarrollo de determinadas actividades de aventura. Como resultado, el territorio se encuentra en posición de adoptar estrategias Ofensivas; estas son las de mayor impacto, las cuales se basan en aprovechar en mayor medida las oportunidades a través de las fortalezas.

\section{Propuesta de desarrollo.}

\section{Nivel I: Desarrollo y Lanzamiento del Producto Turístico.}

Concepto de producto-destino y ofertas actuales: Se analiza la Oferta General de las Agencias visitadas, así como el tipo de productos on-request que diseñan. Se toma como referencia de estudio la oferta real que existe en el territorio Habana-Matanzas para realizar el inventario de los atractivos turísticos que se encuentran en explotación. Este se efectuó con la Empresa 
Nacional para la Protección de la Flora y la Fauna, sobre los recursos que se encuentran en dicho territorio.

Análisis General: Se selecciona como área de estudio el territorio de la Llanura HabanaMatanzas correspondiente a las provincias de La Habana y Mayabeque. De esta forma, se logra mejor administración de los territorios para la actividad turística, y evitar la saturación de las áreas geográficas. Se debe tener en cuenta que las seleccionadas pertenecen al Gobierno Municipal; no son administradas por la Empresa Nacional para la Protección de la Flora y la Fauna o alguna otra relacionada al desarrollo de las áreas.

Sobre el territorio objeto de estudio en la provincia de Mayabeque, en sentido general, y que constituye entonces la competencia más próxima a los productos que se diseñan, cabe destacar que la actividad turística de la provincia se desarrolla en Santa Cruz del Norte. Para los vacacionistas, la parte más llamativa del litoral son las playas situadas a unos $6 \mathrm{~km}$ de dicho poblado, y abarcan desde el río Jibacoa hasta Boca de Canasí para conformar una franja de $9,5 \mathrm{~km}$ de pequeñas y magníficas riberas situadas al interior de caletas, coronadas por alturas y colinas pobladas de tupida vegetación. Dado que este es el polo asociado al desarrollo turístico en la provincia, se realiza el estudio de la zona seleccionada, aledaña al mismo. La actividad en los demás municipios de Mayabeque resulta incipiente, para lo cual se hace necesario el estudio de los recursos con oportunidad de éxito para las actividades de aventura. Esto ha derivado en la selección de los municipios San José de Las Lajas y Melena del Sur para la puesta en explotación de los productos. De esta manera, se toma en cuenta los niveles de saturación de las áreas, así como el hecho de que se encuentran de formas consecutivas y comunicadas por la autopista La Habana-Melena. El municipio San José de Las Lajas es hoy la cabecera de uno de los territorios más desarrollados de Cuba y la capital económica, deportiva y científica de la provincia Mayabeque. Sin embargo, no se evidencia un desarrollo considerable en uno de los sectores más importantes de la Economía cubana actual, el Turismo. En el caso de Melena del Sur, este es uno de los municipios menos desarrollados, se encuentra consecutivo a San José de Las Lajas y posee recursos naturales que no se encuentran en explotación y son adecuados para la Propuesta de Desarrollo que se diseña.

Para la selección de ideas en la creación de productos turísticos se tomó en cuenta la valoración de las alternativas responsables para con el medio ambiente, teniendo en cuenta que ciertos deportes del Turismo de Aventura, como los que requieren equipamiento de vuelo motorizado, pueden causar agresión sonora al medio, solo por citar un ejemplo; el reto fundamental lo constituye el aprovechamiento de los recursos. Se ha de tener en cuenta que se realizó el estudio de los factores sobre la protección de las áreas con la Empresa Nacional para la Protección del Medio Ambiente. Una vez diseñados a partir de las posibilidades que ofrece el entorno, se evaluó la factibilidad ambiental con dicha Empresa. Se determinó que las actividades seleccionadas cumplen con los indicadores de responsabilidad ambiental que 
se manejan. En este caso se toma como referencia las Fichas de Productos de La Empresa Nacional para la Protección de la Flora y la Fauna. Igualmente, se realiza la revisión de Certificados emitidos por el CITMA para la explotación turística.

Segmento de demanda: Se tiene en cuenta los principales mercados emisores del destino, según el Anuario Estadístico de Cuba; se valoraron los principales segmentos de productos de Turismo de Aventura de la Agencia de Viajes ECOTUR S.A. S.A. Como común denominador resultan los mercados alemán, español y francés. Dentro de estos, los millennials que, según (Burkhard, 2017), serán el mercado más grande de viajes durante los próximos años, siendo estos, en sentido general, así como base para la presente investigación, el centro de decisión de las tendencias dominantes en años venideros.

Canales de Distribución: Se realizará a través de ECOTUR S.A. y las Agencias de Viajes que hoy son sus principales clientes (Cubatur, Havanatur, Cubanacan Ventas Internacionales y Gaviota Tours), con lo cual ECOTUR S.A., con lo que ganaría una comisión entre un 15 y un 20\%. Igualmente, mediante TT.OO. propios como son Destino World, Cubautrement, Aventoura, SKEDIO, Profil Cuba Reisen, Tropik Vedeta, Daiquiri Tour, Cuba for Travel Latitud Cuba, La Coronación, Tiambo Divitur, Top Caribe, Huwans, ACUC y otras agencias extranjeras que solicitan los servicios vía internet; el pago lo realizan a través de transferencias antes de la fecha de ejecución del servicio.

Comunicación Promocional: Se realizará fundamentalmente a través de los medios de Comunicación de la Agencia de Viajes ECOTUR S.A., los cuales son el sitio web de la Agencia, el sitio Web de Cuba Travel, así como los medios de promoción de las agencias con las que tiene contratos.

\section{Propuestas de conceptos de productos:}

\section{Producto 1: Recorrido en Motos Clásicas.}

La Categoría de este producto es "sendero" y la Especialidad, "Observación de Flora, Fauna y Cultura Local". Este pretende mostrar, desde un punto de vista diferente, la riqueza y valor paisajístico del territorio; así como el acercamiento a las costumbres de las localidades. Se podrá observar las Cuevas del Cura y el río Almendares, que nace en las Lomas de Tapaste y desemboca en la Chorrera. Se visitará el poblado de San José de Las Lajas y se realizará una parada en "El Pino", restaurante donde degustarán un almuerzo basado en comida criolla fundamentalmente. El área de Influencia será entre San Francisco Javier y las Lomas de Tapaste. San Francisco Javier es una loma extensa y de cumbre extendida al norte de Tapaste. Tapaste son lomas al norte del municipio pertenecientes a la serie central del grupo de La Habana Matanzas. El recorrido se realizará por las carreteras que conectan dichos puntos geográficos. La Administración será de las Autoridades Locales. Los Guías Especializados 
serán contratados con la Agencia Hard Road, chilena, que trabaja actualmente con la Agencia de Viajes Havanatur.

\section{Producto 2: Motos enduro.}

Se trasladará a los turistas desde la Estación San José hasta la entrada de los Campos de La Jaula, a partir de donde se iniciará el recorrido. Este posee un sendero natural y accidentado sobre suelos pardos. Se puede apreciar la flora del lugar, que tiene como exponentes simbólicos el árbol y la flor del Copey; y la fauna, que tiene como exponentes simbólicos la jutía y el majá de Santamaría. El área de influencia será Los Campos de La Jaula en San José de Las Lajas. La Administración pertenecerá a las Autoridades Locales. Los Guías Especializados serán garantizados por la Agencia de Viajes Havanatur.

\section{Producto 3: Cicloturismo en llanuras accidentadas y zonas urbanas.}

Este producto es una combinación de viajes en bus con cicloturismo y visitando lugares de interés local, nacional y mundial. Cuenta con guías de turismo especializados en su trabajo, conocimientos de primeros auxilios y mecánica, con un vehículo de soporte (bus) todo el tiempo. La zona se caracteriza por la presencia de alturas y llanuras onduladas. El municipio presenta un relieve uniforme donde no existen elevaciones que superan $15 \mathrm{~m}$ de altura desde el nivel del mar, por lo cual, la ruta es apta para personas con condiciones físicas favorables sin ser demasiado estrictos. El área de influencia será el municipio San José de Las Lajas, desde Pedro Pi, en el cual se encuentran llanuras accidentadas y rodeadas de lomas de escasa elevación al sur, como las de Camoa, Carmen, Chávez y otras formando grupitos independientes, así como al norte, elevaciones más notorias como las Lomas de Tapaste. La localización de esta actividad sería, entonces, más hacia el Sur, que el recorrido de las motos clásicas. La Administración pertenecerá a las Autoridades Locales. Los guías especializados son garantizados por la Agencia de Viajes ECOTUR S.A., que serán Licenciados en Biología y Geografía, Ingeniero y Técnico Forestal.

Regulaciones Específicas para los Productos 1, 2 y 3: No se admiten más de 6 vehículos al mismo tiempo en una ruta para las motos y 12 para las bicicletas. La cantidad de Guías por grupo será 1. La frecuencia de visitas será 1 al día. La visita solo se admite con el servicio de guías especializados, que trasmitirán al cliente el código de conducta. El recorrido solo se hará en las vías señalizadas. Se llevará a los depósitos de basura señalizados todos los desechos. No se permite extraer elementos del medio natural, ya sean bióticos o abióticos; así como no causar daños ni molestias a la flora y la fauna silvestre del lugar, ni a los animales domésticos vinculados a la comunidad. Se debe fumar solo en lugares indicados y ser respetuosos en la toma de fotografías, filmaciones o interactuar con la comunidad local. 


\section{Producto 4: Bungee Jumping desde Globo Aerostático, en los Mogotes de Barney.}

Este se realizará desde el propio globo aerostático, debido a que el territorio es de llanura y no existe montaña con altura considerable para ello. Se descarta el despeñamiento desde helicópteros, en aras de evitar los conflictos medioambientales por agresiones sonoras. El área de influencia será los Mogotes de Barney, San José de Las Lajas. La Administración pertenecerá a las Autoridades locales. Los guías especializados serán garantizados por la Agencia de Viajes ECOTUR S.A. Esta práctica presenta Regulaciones específicas. No se admiten más de 3 personas en el globo, teniendo en cuenta la carga del equipamiento necesario para la práctica de Bungee Jumping; la frecuencia de Vuelo es de 4 al día; el vuelo solo se admite con el guía especializado; se prohíbe causar daños ni molestias a la flora y la fauna silvestre. Se debe ser respetuosos en la toma de fotografía y filmaciones; realizar periódicamente revisiones técnicas del equipo de vuelo, siguiendo los manuales de vuelo establecidos; asegurar la licencia de transporte aéreo comercial del piloto del globo aerostático, avalado por alguna agencia estatal de seguridad aérea y con licencia homologada; cumplir con todas las normas de seguridad aérea del país; disponer de todos los equipos tecnológicos para la navegación aérea (altímetro, varímetro -mide la velocidad vertical media-, termómetro, brújula, aparato de radio y extintor -de carácter obligarorio-); garantizar la tenencia del seguro de responsabilidad civil y a pasajeros que cubra todo tipo de riesgos. Se prohíbe el vuelo a embarazadas, personas con obesidad (más de $120 \mathrm{~kg}$ ), niños menores de 6 años y personas que sufran algún tipo de dolencia cardíaca o vértigo.

\section{Producto 5: Kayak en Río Mayabeque con autocaravanas para el traslado.}

Esta práctica no se ve limitada en relación con las corrientes de los ríos; estos se clasifican según su dificultad, y todos son aceptables para la práctica del mismo. Se desarrollará la actividad sobre una distancia aproximada de $1 \mathrm{~km}$. El área de Influencia será el río Mayabeque, en el territorio que comprende el municipio San José de Las Lajas y desemboca en la playa del mismo nombre. El Río Mayabeque Antiguo, seleccionado para la actividad, desemboca en la playa del mismo nombre; posee una extensión de más de $10 \mathrm{~km}$ y se forma por la Unión de los Ríos La Luisa y La Teresa; tiene alrededor de 150 curvas y más de 100 pequeños manantiales. La Administración pertenecerá a las Autoridades Locales. Los guías especializados serán garantizados por el Grupo Marina Marlin. Este presenta Regulaciones específicas. Los guías especializados trasmitirán al cliente el código de conducta; el recorrido solo se admite con la presencia de guías especializados; no se permite extraer elementos del medio natural, ya sean bióticos o abióticos; no causar daños ni molestias a la flora y la fauna silvestre del lugar. 


\section{Producto 6: Kitesurf y Waterskiing con uso de autocaravanas para el traslado.}

Para el waterskiing, las condiciones resultan compatibles, en tanto, es suficiente con que el agua esté tranquila y el espacio en el cual se practica sea amplio. El kitesurf es una disciplina adaptable a las condiciones: con poco viento, en locaciones diversas, solo se debe tener en cuenta qué tipo de cometa usar. Es menester conocer que la dirección del viento es determinante, además, saber que no todas las direcciones del viento son grandes aliadas. Estas actividades se realizarán en el mismo espacio geográfico, en aras de diversificar las opciones en el mismo. Se incluye el uso de autocaravanas para evitar la creación de infraestructura que represente daño al medio ambiente el cliente pueda pasar el día disfrutando de las actividades y maravillas del clima, ya que poseen las comodidades necesarias y alimentación. El área de influencia será Playa Mayabeque. La Administración pertenecerá a las Autoridades Locales. Los guías especializados serán garantizados por el Grupo Marlin S.A. Este presenta Regulaciones específicas. No se admiten más de 4 caravanas al mismo tiempo; la cantidad de guías es 1 por cada deporte y un chofer para cada autocaravana; las actividades solo se realizarán con la presencia de un guía; no se debe estacionar las autocaravanas sobre la duna; se debe llevar a los depósitos de basura señalizados todos los desechos.

Acciones inmediatas para la comercialización de los seis productos: Se debe uniformar a los guías; construir y mantener facilidades de infraestructura; dar mantenimiento especializado (limpieza y control de erosión); señalizar las rutas y vías de acceso; mantener limpios de desechos y basuras; hacer cumplir las regulaciones específicas (Responsable: Administración y Cuerpo de Guardabosques); confeccionar el código de conducta (responsable: Administración Local). Para el caso del producto 1, es necesario obtener, del Centro Provincial de Higiene y Epidemiología, la certificación de no existencia de riesgo epidemiológico en las cuevas. Luego, para el producto 4, es preciso comprar del equipamiento.

\section{Desarrollo y Evaluación de conceptos:}

Para la evaluación de los productos, se lleva a cabo el Test de O`Meara. El correspondiente procedimiento aritmético, a partir de las opiniones de los expertos, permite establecer las posibilidades de alcanzar resultados comerciales que representan el éxito. Para este análisis se seleccionaron cinco Expertos, mismos a los que se realizó la entrevista en profundidad. Los factores establecidos para este propósito, y los cuales están presentes en los diferentes productos son: Comercialidad, Durabilidad y Capacidad Productiva. Para cada factor se determinaron sus respectivas variables: relación con las líneas de productos actuales, efectos en las ventas de los productos actuales y facilidad de Comunicación (Comercialidad); amplitud de mercado, resistencia a las fluctuaciones cíclicas y exclusividad de diseño 
(Durabilidad); equipo necesario, conocimiento del producto y personal necesario, y disponibilidad de materias primas (Capacidad Productiva).

Los resultados evidencian el orden de éxito potencial de los productos, a partir del puntaje que obtienen. Se obtiene resultado de Éxito Extraordinario para el Recorrido en Motos Clásicas $(98,88)$ y Bungee Jumping desde Globo Aerostático $(94,12)$. Se valora Éxito Grande para el Kitesurfing y Waterskiing en Playa Mayabeque con uso de autocaravanas $(87,72)$, recorrido con Motos Enduro $(85,48)$, Kayak en Río Mayabeque con uso de autocaravanas $(84,24)$ y Cicloturismo $(80,00)$.

\section{Nivel II: Estudio de Oportunidad.}

Se realiza el Estudio de la viabilidad comercial, técnica, organizativa y económicofinanciera. De ello, se extrae el resumen de aspectos fundamentales:

Comercialización: ECOTUR S.A. posee una página web (www.ECOTUR S.A.cuba.tur.cu) a través de la cual se puede acceder a sus productos; asimismo, se encuentra en el proceso de inserción de una pasarela de pago, que se proyecta a ser implementado en el corto plazo. Por su parte, la Agencia Cubatur se encuentra en proceso de inserción de una pasarela de pago en el mediano plazo, no de forma directa a través de su página web, sino como un link que será compartido en la misma.

Servicios a contratar: La Agencia de Viajes ECOTUR S.A. dispone del equipamiento necesario para el desarrollo de las actividades. Para los servicios de traslado de Gaviota Tours Cuba, esta dispone de los ómnibus de la empresa transportista Transgaviota, para clientes en transfer colectivos y privados, así como Transtur. La Agencia Gaviota Tours S.A. posee facilidades tecnológicas y know how para la instalación de oficinas, renta de servicios de comunicación, trámites migratorios, jurídicos y renta de transportación. Específicamente para los recorridos en motos clásicas y enduro, vale realizar el contrato con la Agencia Hard Road, chilena, que tiene contrato actualmente con la Agencia de Viajes Havanatur S.A. Sin embargo, la Agencia Havanatur S.A., no debe ser necesariamente un intermediario.

Aspectos organizativos: Los productos de Turismo de Naturaleza y Aventura en Cuba deben obtener la Licencia Medioambiental del Ministerio de Ciencia, Tecnología y Medio Ambiente para la explotación del territorio y la Licencia del Ministerio de Turismo. Sobre la Licencia del CITMA, resultan dos obligaciones de carácter general para todos los productos que el titular de la actividad debe imponer al ejecutor las condiciones de esta Licencia Ambiental y delimitar responsabilidades en caso de incumplimiento, así como la copia de la Licencia Ambiental "in sito". El inversionista aceptará el Plan de Monitoreo que será impuesto por la Autoridad Ambiental de acuerdo con la sensibilidad del lugar. 
Se indagó sobre trabajos investigativos similares, en aras de mantener el curso de lo novedoso. (Carrión, 2016), igualmente en respuesta a la necesidad de creación de nuevos productos, estudia las potencialidades del destino Holguín para los productos de naturaleza a partir del rediseño de la Feria Comercial de Turismo de Naturaleza TURNAT 2017; el enfoque de la modalidad es generalizado. (Porras, 2016), realiza una estrategia de desarrollo para el Turismo de Naturaleza en el territorio Villa Clara, a partir del levantamiento de los recursos turísticos, para proponer acciones estratégicas, lo que no implica la creación de productos turísticos. (Salina Chávez, Salinas Chávez, \& Mundet I Cerdan, 2019), como en el presente trabajo, caracterizan con un enfoque geográfico económico la evolución del turismo en Cuba y sus posibilidades de desarrollo; investigan las regiones donde se concentran los principales atractivos, infraestructura e inversiones para el desarrollo, en este caso, del ecoturismo, turismo de aventura y turismo rural; sin embargo, para el que se escribe, constituyó solo el estudio preliminar para la creación de productos de Turismo de Aventura. Luego, este artículo plantea una propuesta para el desarrollo de una zona de categoría geográfica que no se había abordado antes para ello, comprendido en un territorio cuyo desarrollo turístico es incipiente. Se realiza una investigación a cabalidad de los atractivos turísticos, para obtener resultados en forma de productos como propuesta de desarrollo para el Turismo de Aventura específicamente; otras fuentes desarrollan el turismo de Naturaleza en sentido general, lo que puede o no enfatizar en la sub-modalidad mencionada.

\section{Conclusiones:}

El trabajo de investigación del presente proyecto acarrea las siguientes conclusiones:

- El Turismo de Naturaleza en Cuba está fundamentalmente asociado a la actividad turística de sol y playa.

- La actividad turística en Cuba en el destino La Habana enfoca los esfuerzos principalmente en el Turismo Cultural y de Ciudad.

- El desarrollo turístico en la zona enmarcada de la provincia de Mayabeque es incipiente.

- En el territorio seleccionado, se desarrollan actividades aisladas de Turismo de Aventura.

- Existen recursos inutilizados en el territorio objeto de estudio de la Llanura Habana-Matanzas, que constituyen potenciales para el desarrollo de actividades de Turismo de Aventura. 
- Los municipios de San José de las Lajas y Melena del Sur poseen recursos naturales para el desarrollo de la Propuesta de Desarrollo del Turismo de Aventura.

\section{Referencias bibliográficas.}

Aldana, S., Vereda, F., Hidalgo-Alvarez, R., \& de Vicente, J. (2016). Facile synthesis of magnetic agarose microfibers by directed selfassembly. Polymer, 93, 61-64.

Barrado, D., \& Vila, M. (1999). Turismo en espaios de montaña y naturales. En D. Barrado, \& J. Calabuig, Geografía Mundial del Turismo (págs. 123-150). Madrid: Síntesis.

Barrios, E. (2007). Ciclo de Vida de un producto y sus estrategias relacionadas.

Bhat, S., Tripathi, A., \& Kumar, A. (2010). Supermacroprous chitosan-agarose-gelatin cryogels. in vitro characterization and in vivo assesment for cartilage tissue engineering. Journal of the Royal Society Interface, 1-15.

Bossis, G., Marins, J., Kuzhir, P., Volkova, O., \& Zubarev, A. (2015). Functionalized microfibers for field-responsive materials and biological applications. Journal of Intelligent Material Systems and Structures, 1-9.

Burkhard, S. N. (2017). 7 travel trends tht will drive the global tourism industry.

Carrión, M. B. (2016). Rediseño de la Feria Comercial de Turismo de Naturaleza TURNAT, Holguín 2017. Tesis en Opción al Título de Licenciado en Turismo. Facultad de Ingeniería Industrial y Turismo. Holguín.

Cortés, J., Puig, J., Morales , J., \& Mendizábal, E. (2011). Hidrogeles nanoestructurados termosensibles sintetizados mediante polimerización en microemulsión inversa. Revista Mexicana de Ingeniería Química., 10(3), 513-520.

Dias, A., Hussain, A., Marcos, A., \& Roque, A. (2011). A biotechnological perspective on the application of iron oxide magnetic colloids modified with polysaccharides. Biotechnology Advances 29, 29, 142-155.

Estrada Guerrero, R., Lemus Torres, D., Mendoza Anaya, D., \& Rodriguez Lugo, V. (2010). Hidrogeles poliméricos potencialmente aplicables en Agricultura. Revista Iberoamericana de Polímeros, 12(2), 76-87.

García-Cerda, L., Rodríguez-Fernández, O., Betancourt-Galindo, R., Saldívar-Guerrero, R., \& TorresTorres, M. (2003). Síntesis y propiedades de ferrofluidos de magnetita. Superficies y Vacío., $16(1), 28-31$. 
HOSTELTUR. (31 de octubre de 2016). Cuba aún no explota el $85 \%$ de su potencial turístico. España.

IICA Costa Rica. (2018). Guía para la elaboración del Plan de Desarrollo Turístico en un territorio. . Costa Rica.

Ilg, P. (2013). Stimuli-responsive hydrogels cross-linked by magnetic nanoparticles. Soft Matter, 9, 3465-3468.

Lewitus, D., Branch, J., Smith, K., Callegari, G., Kohn, J., \& Neimark, A. (2011). Biohybrid carbon nanotube/agarose fibers for neural tissue engineering. Advanced Functional Materials, 21, 2624-2632.

Lin, Y.-S., Huang, K.-S., Yang, C.-H., Wang, C.-Y., Yang, Y.-S., Hsu, H.-C., . . Tsai, C.-W. (2012). Microfluidic synthesis of microfibers for magnetic-responsive controlled drug release and cell culture. PLOS ONE, 7(3), 1-8.

Lorenzo, T. D. (2016). El Ecoturismo en Cuba: Evolución y perspectivas de futuro. Una interpretación a partir de una comparativa con Costa Rica. Trabajo Final de Grado. Universidad de Girona. Facultad de Turismo. Girona.

Mezquita, J. C., \& Fiallo Rodríguez, J. (2002). Los métodos científicos en las investigaciones pedagógicas. La Habana, Cuba.

Mincetur. (mayo de 2014). Manual para la Planificación de Productos Turísticos.

Perelló, J. L. (2001). Desarrollo y Promoción de Productos Turísticos. Universidad de La Habana. La Habana, Cuba.

Porras, Y. R. (2016). Estrategia de Desarrollo para el Turismo de naturaleza en el destino Villa Clara. Tesis de Diploma. Facultad de Ciencias Económicas. Departamento de Turismo. Santa Clara.

Ruiz Estrada, G. (2004). Desarrollo de un Sistema de liberación de fármacos basado en nanopartículas magnéticas recubiertas con Polietilénglicol para el tratamiento de diferentes enfermedades. Madrid: Universidad Autónoma de Madrid. Departamento de Física Aplicada.

Salina Chávez, E., Salinas Chávez, E., \& Mundet I Cerdan, L. (2019). El Turismo en Cuba: Desarrollo, Retos, Perspectivas. Rosados Ventos. Turismo e Hospitalidade.

Song , J., King, S., Yoon , S., Cho, D., \& Jeong, Y. (2014). Enhanced spinnability of narbon nanotube fibers by surfactant addition. Fiberes and Polymers, 15(4), 762-766. 
Tartaj, P., Morales, M., González-Carreño, T., Veintemillas-Verdaguer, S., \& Serna, C. (2005). Advances in magnetic nanoparticles for biotechnology applications. Journal of Magnetism and Magnetic Materials, 290, 28-34.

Wulff-Pérez , M., Martín-Rodriguez, A., Gálvez-Ruiz, M., \& de Vicente, J. ( 2013 ). The effect of polymer surfactant on the rheological properties of nanoemulsions. Colloid and Polymer Science, 291, 709-716.

Zamora Mora, V., Soares, P., Echeverria, C., Hernández , R., \& Mijangos, C. (2015). Composite chitosan/Agarose ferrogels for potential applications in magnetic hyperethermia. Gels., 1, 69-80. 


\section{PARA CITAR EL ARTÍCULO INDEXADO.}

Saumell Lafargue , C., \& Hernández Rodríguez , G. C. (2020). Propuesta de Desarrollo para el Turismo de Aventura en el territorio de la Llanura Habana-Matanzas en los límites de las provincias La Habana y Mayabeque. Explorador Digital,4(3), 140-158. https://doi.org/10.33262/exploradordigital.v4i3.1314

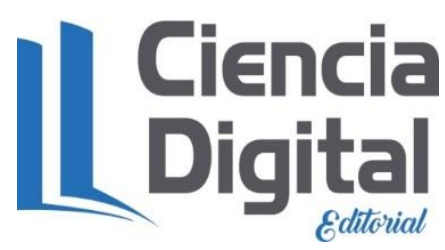

El artículo que se publica es de exclusiva responsabilidad de los autores y no necesariamente reflejan el pensamiento de la Revista Explorador Digital.

El artículo queda en propiedad de la revista y, por tanto, su publicación parcial y/o total en otro medio tiene que ser autorizado por el director de la Revista Explorador Digital.
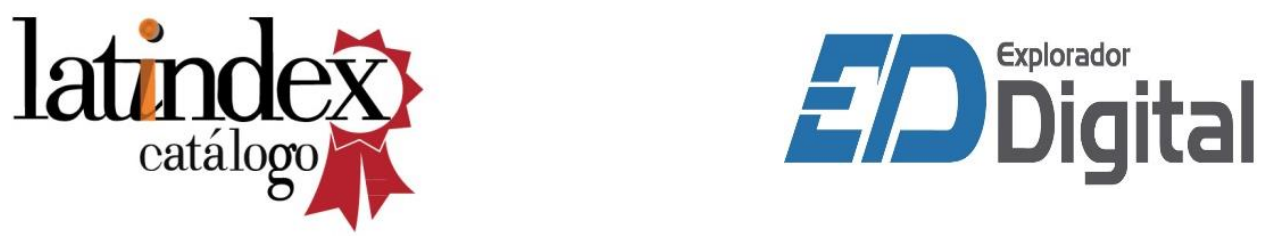